\title{
Étude de la pratique picturale d'un journal engagé
}

\author{
L'usage de l'art dans Le Monde \\ diplomatique
}

Flavie Holzinger

\begin{abstract}
Cet article examine les choix iconographiques du Monde diplomatique, journal à la réputation sérieuse, mais également engagé et militant, politiquement et socialement, dont les buts sont d'alerter l'opinion et susciter le débat public. L'image tient en effet une place particulière dans Le Monde diplomatique puisque c'est par la reproduction d'œuvres d'art que le journal accompagne ses articles. Ainsi, de manière distincte et créative, le mensuel offre à l'art une visibilité singulière. La démarche artistique du Monde diplomatique n'est pas celle d'un jugement esthétique, mais d'un choix qui repose sur le sujet de l'œuvre, sur son titre, ou encore sur le parcours et la nationalité de l'artiste. Le rapport entre écrit et image s'établit par un jeu entre adéquation, décalage et distanciation. Le Monde diplomatique donne à l'image une place égale au texte, l'art participant ainsi au sens du discours du journal. En tant qu'acteur critique dans le débat public, cette entreprise de presse recrée l'articulation entre art et politique et entre art et démocratie. L'usage de l'art dans Le Monde diplomatique participe à sa volonté de résistance à l'ordre établi sur le fond et sur la forme. Mots-clés: Monde diplomatique, illustration, usage de l'art.
\end{abstract}

\section{Introduction}

En tant qu'acteurs géopolitiques, et à travers les représentations ${ }^{1}$ qu'ils diffusent, les médias influencent l'opinion publique, amènent au débat public et

\footnotetext{
${ }^{1}$ En géopolitique, la représentation est une construction d'idées, plus ou moins logiques ou cohérentes, destinée à décrire une partie de la réalité. La représentation a pour fonction de faire partager des idées et des valeurs à tout un groupe pour l'émouvoir et le mobiliser. (Lacoste, 1993 : 1278)
} 
peuvent même parfois agir sur les points de vue et les décisions des dirigeants. La presse contribue ainsi au débat démocratique. En tant que moyen possible de contestation, elle est un véritable levier d'expression essentiel au fonctionnement de la démocratie. Dans la presse française, Le Monde diplomatique tient une place particulière. C'est un journal d'opinion dont la ligne éditoriale est très ancrée à gauche. Depuis les années 1970, sous l'impulsion de Claude Julien, responsable principal du titre de presse à partir de 1973, la rédaction dénonce l'impérialisme américain et le système capitaliste et libéral. La ligne éditoriale du mensuel d'inspiration tiers-mondiste a largement accompagné le mouvement altermondialiste à la fin des années 1990. Exemple unique dans la presse française, Le Monde diplomatique a fait le choix, il y a plus de vingt ans, d'illustrer ses textes par des reproductions de tableaux modernes et contemporains, voire anciens, par des photos de sculptures ou d'architectures, par des photographies d'auteurs, des photographies conceptuelles ou plasticiennes, ou par des dessins. Ces choix iconographiques sont partie prenante de son identité. Mais quelle est véritablement l'utilisation faite de l'art dans Le Monde diplomatique? Quels sont les outils et les stratégies convoqués par la rédaction pour convaincre et mobiliser ses lecteurs? Comment l'art participe à son objectif militant? L'insertion d'œuvres d'art dans les colonnes du journal constitue-t-elle une preuve de l'efficacité possible de l'art dans un combat politique et social au sein d'une démocratie ? Tout d'abord, une analyse du Monde diplomatique, en tant que journal engagé et militant, est capitale : comment ce journal fondé en 1954 a réussi à rester une référence sérieuse pour une bonne partie de l'opinion publique et notamment pour les enseignants, tout en bénéficiant d'une large diffusion internationale, dans un contexte difficile pour la presse? Il faudra s'interroger, ensuite, sur le statut de l'image d'art dans un mensuel d'information aussi particulier que Le Monde diplomatique pour finir, enfin, par tenter de tisser brièvement le lien entre art et démocratie à travers l'exemple du Monde diplomatique.

\section{Le Monde diplomatique : un média de la démocratie}

\subsection{Un projet engagé}

La presse a un rôle essentiel dans les débats géopolitiques à condition que ce rôle s'exerce dans des États où existe une réelle liberté d'expression. Le discours d'information est la base de la démocratie. On parle souvent de la presse en tant que "quatrième pouvoir», alors qu'elle est considérée comme un contrepouvoir indispensable à l'existence de la démocratie (Lacoste 1993 : 1245). Un organe de presse libre et contestataire est même un des éléments fondamentaux d'une démocratie. Les signes tangibles de progrès de la démocratie dans certains 
pays peuvent se mesurer par l'augmentation du nombre de journaux, par le degré de contrôle qu'exercent les pouvoirs politiques - mais également financiers - sur la presse, et par la liberté d'expression des journalistes, liberté d'expression qui caractérise la rédaction du Monde diplomatique. L'alerte écologique, les inégalités Nord/Sud, la remise en cause du modèle libéral, la contestation de l'unilatéralisme, de l'hégémonisme et de l'impérialisme américains, la critique politique du gouvernement français, la dénonciation du système médiatique corrompu et celle de la surdité des intellectuels, la fascination pour l'Amérique latine, le mouvement de solidarité avec les Palestiniens et l'appel à la résistance contre l'ordre établi sont les thèmes largement développés dans le mensuel. Le journal revendique sa place à contre-courant dans la bataille des idées par rapport aux autres médias. Ses journalistes utilisent régulièrement les grands paradigmes géographiques du Nord et du Sud et légitiment les actions des révolutionnaires du monde entier. Le Monde diplomatique est intéressant à étudier dans la mesure où il se situe au cœur d'un réseau : les idées qui y sont publiées sont reprises par un certain nombre de milieux intellectuels et politiques.

\subsection{Les influences du Monde diplomatique : ses réseaux et sa diffusion}

Le journal est également au cœur d'un réseau d'associations et de comités engagés dont Le Monde diplomatique se fait l'écho en contribuant à promouvoir dans ses colonnes les débats et rencontres. Il entend susciter le débat public, choquer les consciences et alerter l'opinion. Le Monde diplomatique revêt en quelque sorte une fonction politique et sociale. Des groupes de réflexion, comme les Amis du Monde diplomatique ont été créés en 1996. Ces clubs de lecteurs, qui ont la particularité de former, ensemble, les principaux actionnaires du journal, font la promotion du mensuel et organisent différentes conférences, débats, et groupes de discussion. Ils participent à l'image d'un Monde diplomatique actif, indépendant et militant, acteur dans le débat démocratique. Le journal est l'instigateur de plusieurs associations proches de l'altermondialisme. L'idée de créer l'Association pour la taxation des transactions financières pour l'aide aux citoyens, Attac, ainsi que celle de créer un Forum social mondial et un Observatoire international des médias sont nées au siège du journal grâce à des édito- 
riaux d'Ignacio Ramonet ${ }^{2}$. Le mensuel se place à contre-courant de ce qui se fait actuellement dans le champ médiatique. Il a en effet échappé à la concentration au sein de grands groupes de presse en ayant réussi sa filiation ${ }^{3}$ et s'est distingué par une ligne éditoriale nettement progressiste, voire tiers-mondiste. En mars 2011, le mensuel comptait quatre-vingt-quatre éditions internationales en vingt-sept langues ${ }^{4}$ : quarante et une imprimées et quarante-trois électroniques pour un tirage total de 2,4 millions d'exemplaires ${ }^{5}$ à travers le monde selon la rédaction du mensuel. Cette forte présence à l'étranger a représenté un vecteur de communication dans le réseau altermondialiste et a pu ainsi constituer un bon levier permettant de sensibiliser des individus et des groupes à l'échelle planétaire. Le Monde diplomatique revendiquait 242403 lecteurs pour son édition française à son apogée en 2003. Dans un contexte difficile pour la presse, le journal a ensuite subi plusieurs années de perte. En 2010, la diffusion France payée du Monde diplomatique a été de 123983 exemplaires selon l'OJD, l'Office de justification de la diffusion.

\subsection{Un combat pour une presse libre et indépendante}

La liberté d'expression du Monde diplomatique se manifeste également par son militantisme informationnel et sa lutte contre une pensée unique. Le principe du militantisme informationnel est de mettre en œuvre ses propres dispositifs de production d'information pour effectuer une critique des médias : une critique externe du pouvoir médiatique et de la globalisation des industries de l'information et une critique interne du travail de journaliste. Cette logique rend responsables les médias de la mauvaise hiérarchisation des priorités de l'agenda public et les accuse de céder aux pressions des lobbys industriels, de reproduire

\footnotetext{
${ }^{2}$ L'éditorial «Désarmer les marchés» de décembre 1997 marque les débuts d'Attac. L'éditorial « Le cinquième pouvoir » (octobre 2003) suggère la formation d'un Observatoire international des médias.

${ }^{3}$ En 1996, Le Monde diplomatique devient, à sa demande, une société à part entière, filiale du Monde qui conserve $51 \%$ de son capital. Le reste est partagé entre l'association des Amis du Monde diplomatique (25\%) et celle des salariés du mensuel (24\%). En plus de sa liberté rédactionnelle, Le Monde diplomatique a ainsi conquis son indépendance légale.

${ }^{4}$ Allemand, anglais, arabe, arménien, bulgare, biélorusse, chinois, coréen, croate, espagnol, espéranto, farsi, finlandais, grec, italien, japonais, kurde, norvégien, polonais, portugais, roumain, russe, slovaque, slovène, suédois, tchèque, turc.

${ }^{5}$ Informations disponibles sur http://www.monde-diplomatique.fr/int/ Page consultée le 14 février 2011.
} 
sans réserve les discours dominants des gouvernants et des institutions internationales, de participer ainsi à la production d'une pensée unique et de marginaliser les propositions alternatives (Cardon \& Granjon 2003). Cette critique dénonce la propagande de la globalisation que martèleraient les médias à large audience. Le militantisme informationnel appelle à la création d'un contrepouvoir critique pour lutter contre l'allégeance des entreprises de presse au monde politico-économique. Le Monde diplomatique appelle donc à un contremodèle d'information, qui se réclame d'un travail scientifique: référence à l'exactitude et à la vérité, à la liberté, l'indépendance et la rigueur. Le Monde diplomatique est bien un journal politisé, engagé et militant.

Dans ce contexte, quelle est la place de l'image dans Le Monde diplomatique? Première particularité : cet organe militant dit refuser la publicité, ou du moins la limiter officiellement à $5 \%$, au maximum, de ses recettes. Par ailleurs, le caractère résolument international du journal nécessite l'utilisation d'une cartographie remarquable. Les cartes publiées dans le mensuel sont abondamment reprises dans les milieux universitaires et sur le média Internet. Elles représentent l'un des piliers de l'information et des idées diffusées par le journal. Des atlas, publiés régulièrement, font partie de l'entreprise de diversification des publications ${ }^{6}$ entreprise par la SA Le Monde diplomatique. La carte géographique n'est pas objective, elle dépend de ce que ses auteurs décident d'y faire figurer, ce qu'ils veulent souligner, mettre en valeur ou bien ignorer. Premier constat sur la place de l'image dans le mensuel : la limitation de la publicité et les cartes publiées participent au caractère engagé et militant du journal.

\section{Le Monde diplomatique : une identité visuelle unique dans la presse}

\subsection{Une logique médiatique en mouvement : l'expression par l'image}

Mais ce qui nous intéresse ici est une autre complémentarité entre image et écrit dans le domaine de l'information. La construction du message informatif a beaucoup évolué depuis les débuts de la presse écrite. De manière générale, les journaux ont réduit la part des textes pour laisser une place croissante aux pho-

\footnotetext{
${ }^{6}$ Le bimestriel Manière de voir, la coédition de livres, les CD-Rom permettant la consultation des archives du journal, les atlas, les hors-séries, une bande dessinée.
} 
tographies. L'actualité a été scénarisée, la photographie tenant le rôle principal et l'écrit le second. Cette priorité donnée à la photographie impose au rédacteur, qui doit tenir compte des informations véhiculées par les photographies, une nouvelle méthode d'écriture (Mouriquand 1999 : 22). Le directeur artistique, ou celui qui conçoit le visuel, est devenu un acteur majeur des rédactions, et en particulier pour la presse magazine. Le succès de la visualisation de l'information a donné naissance à une nouvelle discipline, l'infographie, qui mélange dessins et quelques mots-clés dans un travail de synthèse. Tout article est accompagné d'une série de repères péritextuels (comme les titres, les intertitres, les images, les légendes, etc.) qui participent au «contrat médiatique » (Charaudeau, 1997), qui est composé de deux visées, la visée d’information et la visée de captation. Sur les chemins de $\mathrm{fer}^{7} \mathrm{du}$ Monde diplomatique, on constate un nombre important d'hyperstructures, tel que les a étudiées Gilles Lugrin $(2001)^{8}$, c'est-à-dire un ensemble rédactionnel qui regroupe des articles de genres différents dans des unités rédactionnelles clairement distinguées (sur une demi-page, une page entière, ou deux pages symétriques). En effet, les spécialistes des médias s'accordent pour reconnaître deux tendances majeures dans l'évolution de la presse écrite : l'éclatement des articles en modules plus courts - ce qui n'est pas le cas du Monde diplomatique, qui se place ici à contre-courant, les articles restant denses et la place accordée au sujet plus conséquente que dans la plupart des autres journaux d'information - et un développement du visuel - que ce soit au niveau de la mise en page ou de l'infographie. C'est vrai aussi pour Le Monde diplomatique qui, on l'a vu, conçoit régulièrement des cartes et publie (nous allons y venir) des œuvres d'art pour illustrer les sujets traités. Les articles, les bibliographies, les brèves, les chronologies, les encadrés, les extraits et les points de repère peuvent également alimenter l'hyperstructure, laissant ainsi apparaittre une hétérogénéité globale qui se décline autour d'une unité thématique commune. L'hyperstructure correspond à une tendance générale de la presse à favoriser une lecture visuelle de l'information. Son développement dans la presse écrite et magazine semble d'ailleurs correspondre à celui des nouvelles technologies de l'information et à celui des techniques de publication assistée par ordinateur. Les pages HTML ont eu pour conséquence de modifier la présentation des informations dans le journal et la pratique journalistique ellemême. Si la presse a eu une forte influence sur la création des premiers sites In-

\footnotetext{
${ }^{7}$ Le chemin de fer est, dans l'édition et la presse écrite, la représentation d'un ouvrage ou d'un journal, page par page et dans sa totalité.

${ }^{8}$ C'est E.-U. Grosse et E. Seibold (1996) qui ont été les premiers à avancer le terme d'hyperstructure.
} 
ternet, il semble aujourd'hui que la tendance s'inverse (Lugrin 2001). Le multimédia favorise une écriture et une lecture non linéaires. Si face à la concurrence d'Internet et à sa baisse des ventes, Le Monde diplomatique n'a pas fait le choix de réduire la longueur de ses articles, sa rédaction s'appuie par contre maintenant largement sur l'infographie. Cependant, par sa nature, cette dernière diffère considérablement de celle des autres journaux.

\subsection{L'expérience de la communication par l'art}

Dans le livre d'art paru à l'occasion des cinquante ans du Monde diplomatique, Ignacio Ramonet raconte que la publication d'œuvres d'art dans les colonnes du journal répond à la volonté de recourir à une iconographie d' "une manière distincte, plus créative ", qui, au contraire du texte, "s'offre d'emblée d'un seul coup, comme une révélation [...] sans exiger, a priori, nul protocole d'approche, aucun déchiffrement particulier» (Ramonet 1994). À l'inverse des autres titres de presse qui illustrent leurs articles par des photographies, des graphiques, des croquis de presse ou des caricatures, Le Monde diplomatique choisit des œuvres d'art pour accompagner ses propos. Par ce biais, la rédaction du Monde diplomatique offre à l'image « une position de plus grande égalité avec le mot» et «à ses créatrices et créateurs (peintres, dessinateurs, photographes, plasticiens), un statut semblable à celui des auteur(e)s des textes » (Ramonet 1994). Mais cela n'a pas toujours été le cas. Lorsque Hubert Beuve-Méry crée Le Monde diplomatique en 1954, il est hostile à la présence des images (Le Monde qu'il lance en 1944 n'en contient pas) mais décide d'illustrer le mensuel de quelques photos. Pendant des années, l'iconographie tient une place qu'on pourrait qualifier d'académique dans le mensuel. Il faut attendre 1989 et l'arrivée de la couleur pour que s'opère le changement. L'entreprise est confiée à Solange Brand. Elle a été iconographe, maquettiste et directrice artistique du Monde diplomatique de 1980 à 2004. Cette responsable du visuel s'est impliquée au sein de la rédaction et dans le rapport particulier et très personnel que cette dernière a établi avec l'image. En 1965, à peine âgée de vingt ans, Solange Brand part pour Pékin où l'attend un poste de secrétaire à l'ambassade de France. Le Général de Gaulle vient alors de reconnaitre la République populaire de Chine. Elle se trouve là confrontée à un autre monde. De manière candide, elle photographie en couleur ce qui s'offre à elle. De cette expérience, Solange Brand publiera un livre en 2005, «Pékin 1966 : petites histoires de la révolution culturelle ", et une exposition itinérante suivra. De retour en France, elle a été secrétaire puis secrétaire de rédaction au Monde dans les années 1970 avant de rejoindre Le Monde diplomatique, alors dirigé par Claude Julien. Au début des années 1980, elle ouvre le mensuel à la photo et aux gravures. 1989 est une année charnière avec la mise en place de rotatives offset qui permettent le recours à la 
couleur et donc l'ouverture à la peinture. L'art accompagne alors l'information politique de manière innovante. Solange Brand n'avait alors aucune théorie ou connaissance pointue de la peinture ; elle s'est surtout imprégnée de l'identité forte du journal tout en se constituant une culture de l'image. Elle a monté une véritable photothèque et est entrée en rapports directs avec les artistes. Sa mission: monter toutes les pages du Monde diplomatique. Elle a donné à l'iconographie une place très différente de celle qu'elle a dans le reste de la presse. Le choix de Claude Julien était de refuser une iconographie purement illustrative et décorative soumise au texte, préférant une image qui dialogue avec ce dernier, offrant à la fois une respiration et une réflexion. Pas d'immédiateté les images ne décrivent pas un fait, mais elles sont recherchées et choisies en fonction du fait qu'elles incarnent le propos en tant que témoignage d'une autre idée; pas d'émotion, pas de redondance ; les images ne doivent pas répéter ni illustrer ce que dit le texte mais le dire autrement.

L'intérêt ici n'est pas de décrire et commenter le contenu de l'ensemble des œuvres publiées dans Le Monde diplomatique. En revanche, à travers quelques exemples, il s'agit davantage de comprendre la vision globale de l'usage de l'art dans le mensuel. Nous n'analyserons donc pas précisément les œuvres mais nous essayerons plutôt de mettre à jour la démarche artistique du Monde diplomatique au vu de son projet militant. Quel est son système de choix ? Les jugements de valeur du directeur artistique ne concernent pas seulement un jugement esthétique. Ils peuvent aussi s'exprimer par le sujet de l'œuvre, ou par son titre, ou encore par le parcours ou la nationalité de l'artiste. Reste à savoir aussi si en s'appropriant l'œuvre et en s'accommodant de la liberté du sens porté par une œuvre, Le Monde diplomatique respecte bien l'art en question ou du moins son idée.

\section{3. Écrit et image d'art : un jeu entre adéquation, décalage et distanciation}

On l'a vu, la ligne éditoriale du Monde diplomatique a, de fait, inspiré un mouvement altermondialiste. L'association Attac, dont l'enjeu premier était de plaider en faveur d'une taxe sur les transactions financières mondiales et de critiquer la logique économique et sociale de la mondialisation néolibérale et des institutions économiques internationales, est née directement de l'éditorial «Désarmer les marchés » (Ramonet, décembre 1997). Ce texte est accompagné d'une peinture de Francis Bacon, «Figure en mouvement» (1985) sur laquelle on voit deux figures humaines, floues, tordues et triturées, en lutte face à une menace et au désordre. Il semble que les figures du peintre irlandais répondent au besoin de rendre visibles des forces invisibles. La déformation de l'apparence humaine 
rend compte du mouvement, de la force, de la pression, et de la réalité de l'individu. L'adéquation entre le texte et l'œuvre semble parfaite.

En février 1991, actualité oblige, Le Monde diplomatique traite de la Guerre du Golfe. L'éditorial de Claude Julien, « une guerre si propre » et l'article d'Ignacio Ramonet, "Gagner la paix », placés en première page du mensuel, sont accompagnés d'une toile de René Magritte, «Les Fanatiques », datée de 1945 et de la représentation d'une huile sur toile, «Puissance aveugle » de Rudolf Schlichter, datée de 1937. Sur la première oeuvre, figure, sur un fond très sombre et menaçant, une flamme flamboyante et violente entourée d'un cercle de pierre, audessus de laquelle est juxtaposé un oiseau de proie, allusion à l'alchimie et, selon la légende, au phénix qui renait de ses cendres. La seconde œuvre du peintre allemand Rudolf Schlichter, représentant les yeux voilés d'un heaume, un soldat géant et casqué qui porte en son sein les désastres de la désolation, fait partie de ses dix-sept œuvres confisquées par les nazis. Ces deux œuvres, dont le contexte est pour l'une l'après-guerre, et pour l'autre la montée du nazisme en $\mathrm{Al}$ lemagne, illustrent dans Le Monde diplomatique le désarroi de la rédaction du journal face à une guerre d'Irak incomprise.

Dans Le Monde diplomatique, le rapport entre l'écrit et l'image est établi selon les cas par l'adéquation mais aussi le décalage ou la distanciation. En mai 2000, Ignacio Ramonet signe un article "Un délicieux despotisme », dans lequel il dénonce le projet impérialiste des États-Unis. Ce texte est accompagné d'une œuvre de Karel Appel qui a pour titre "Une souris sur la table». Une des sources d'inspiration de cet artiste néerlandais est l'art enfantin. Dans son œuvre, Karel Appel s'est attaché à retranscrire les sentiments violents que lui inspire le monde et à se révolter contre les conventions picturales. Résultat: une œuvre colorée d'une grande intensité expressive mais qui, a priori au moins, tranche avec le texte juxtaposé.

Les critères de sélection d'une œuvre pour accompagner un article ne semblent pas se limiter au contenu des œuvres mais peuvent être étendus à l'appartenance de l'œuvre à tel ou tel mouvement artistique. En mars 1998, des photographies de la plasticienne Sophie Ristelhueber, intitulées "Faits », illustrent un dossier intitulé «L'Hégémonie des États-Unis à l'épreuve ». L'artiste s'est rendue dans le désert du Koweït en octobre 1991 pour réaliser des photographies des "territoires cicatrisés ». Elle a pris des vues aériennes et des vues au sol des champs de bataille dans le désert dévasté. Ici, Le Monde diplomatique utilise des photographies des cicatrices de la Guerre du Golfe pour sensibiliser en 1998 le lecteur à l'attitude belliqueuse des États-Unis qui ont comme objectif officiel l'anéantissement des armes de destructions massives irakiennes, et, déjà en arrière-plan selon les journalistes du mensuel, le renversement du président Saddam Hussein. 
En novembre 2001, au lendemain du 11 septembre, Le Monde diplomatique a choisi de traiter l'événement non pas en proposant au lecteur une énième photo des avions détournés détruisant les tours jumelles du World Trade Center de New York mais en publiant trois œuvres de l'artiste Ruth Thorne-Thomsen. Ces dernières s'inscrivent dans le cadre de la série « Mythologies » et constituent de petits théâtres d'images inventés et photographiés par une camera oscura confectionnée dans une boîte à cigares. Ce choix de ne pas montrer la catastrophe ou ses ruines participe à la volonté du Monde diplomatique de ne pas dramatiser davantage l'événement mais de se concentrer sur ses causes.

Dès lors, on peut se demander s'il s'agit d'œuvres d'artistes engagés ou d'œuvres dont le potentiel d'efficacité ou d'action est activé par l'usage qu'en fait Le Monde diplomatique. Dans le journal, «l'image n'illustre pas mais participe du sens ${ }^{9}$. Les œuvres choisies sont différentes du point de vue des styles et des genres. Le Monde diplomatique parie avant tout sur la capacité à exprimer des artistes, sur une communicabilité universelle des œuvres et sur l'intuition sensible de son lectorat. Le directeur artistique de ce journal réalise ainsi des associations d'auteurs et d'artistes autour d'un thème. Les images, pourtant déconnectées de l'actualité, renvoient au réel avec parfois beaucoup d'ironie et d'humour.

En novembre 2003, l'article «Les médias, gardiens de l'ordre social» est accompagné d'une photographie de Cyril Perrin intitulée «Presse purée ». Le titre est explicite : l'artiste a réellement photographié la grille de l'ustensile de cuisine destiné à écraser des légumes. Mais dans presse-purée, il y a presse ainsi que les idées d'écrasement, de déformation et de pression. La rédaction du Monde diplomatique reconnait elle-même dans le livre sur l'art publié pour son cinquantième anniversaire cette interrogation : "Cyril Perrin pensait-il aux médias en photographiant son presse-purée ?».

\subsection{Les éditoriaux : l'art comme arme de persuasion et de mobilisation}

L'art abstrait a sa place dans les colonnes du mensuel. Il a en effet souvent accompagné les éditoriaux d'Ignacio Ramonet entre 1991 et 2008, dates pendant lesquelles ce dernier est à la tête de la rédaction. L'art abstrait a la particularité

\footnotetext{
${ }^{9}$ Présentation de l'ouvrage, Le Monde diplomatique 50, sur le site Internet du Monde diplomatique. Page consultée le 8 mars 2011. http://www.monde-diplomatique.fr /livre/cinquantenaire/
} 
de pouvoir facilement se détacher du réel pour tendre vers la transmission d'un message universel. Rappelons ici l'importance des éditoriaux : l'éditorial est sans doute le genre par excellence de l'article d'opinion. Prenant position sur un fait d'actualité, il engage la responsabilité du journal au point que sa signature peut ne pas être nécessaire. L'éditorialiste jouit ainsi d'une position prestigieuse au sein de la rédaction. L'éditorial semble combiner une exigence de visibilité - sa place est en première page - et d'unicité. Il est partagé entre la séduction et l'argumentation, il se veut subjectif mais se désubjectivise pour incarner les formes de l'opinion du lectorat. L'éditorialiste peut se donner le rôle d'inquiéter ou de rassurer en faisant appel au rationnel ou à l'émotion, au savoir ou à la séduction, et/ou le rôle critique vis-à-vis des pouvoirs politique, économique, scientifique, voire un rôle mobilisateur. Ce rôle critique traduit une volonté d'agir et de mobiliser des lecteurs. Le lecteur doit être séduit ou persuadé, ce qui fait écho à la dimension rhétorique des discours. Patrick Charaudeau (2006) montre que le destinataire d'un discours médiatique peut être envisagé comme cible affective et comme cible intellective. Le lecteur est touché par le jeu sur les émotions provoquées notamment par le lexique, les constructions syntaxiques ou par les visuels. La cible intellective sera intéressée par la lecture des articles qui lui permettront d'établir ou d'occuper une certaine position sociale. Dans le cas du Monde diplomatique, en plus d'être une cible affective et intellective, le lecteur est une cible citoyenne. Il a vocation à se mobiliser.

En janvier 2001, l'éditorial « Porto Alegre», qui annonce le premier Forum social mondial et qui marque le succès du mouvement altermondialiste, est accompagné d'une peinture abstraite d'Auguste Herbin intitulée "Génération » (1959). Cette peinture représente des formes géométriques dont la rigueur du dessin, le jeu des épaisseurs et le rapport entre formes et couleurs donnent une impression de parfait équilibre entre les éléments. Dans la mesure où la peinture abstraite et géométrique se débarrasse de l'objet en se détachant de la représentation figurative, pourquoi la choisir pour illustrer un article aussi engagé ? Les critères esthétiques, la couleur, les lignes, l'équilibre ont pu être convoqués dans le choix, mais peut-être que c'est la part d'imaginaire que l'œuvre suscite qui a été déterminante. De la même manière, en janvier 1996, l'éditorial « Espoir », dans lequel Ignacio Ramonet se félicite du mouvement social de novembre-décembre 1995, est accompagné d'une peinture abstraite géométrique de Geneviève Claisse, «Unité » (1969), qui représente simplement trois formes circulaires et qui comprend trois couleurs. Mais ici, c'est le titre de l'œuvre qui participe au sens de l'image et qui évoque les propos de l'éditorialiste. Le titre de l'œuvre, «Unité», pourrait également être celui de l'article dont voilà les premières lignes : «Par leur formidable révolte sociale de décembre 1995, les Français ont collectivement, exprimé leur refus d'un modèle de société fondé sur l'économisme, le libéralisme intégral, le totalitarisme des 
marchés et la tyrannie de la mondialisation. Ils ont rappelé aux dirigeants un vieux principe républicain : les citoyens préfèrent le désordre à l'injustice. » Le rapport entre écrit et image peut donc s'établir par les légendes. Selon Claude Abastado, en effet, il n'y a pas de messages iconiques purs (Abastado 1980 : 200), le texte participant au sens de l'image. Ainsi, les photographies s'accompagnent souvent d'un commentaire, les tableaux portent un titre.

\subsection{L'origine des artistes : un choix en adéquation avec la ligne éditoriale}

Le Monde diplomatique s'ouvre à d'autres cultures et d'autres modes d'expression en mettant en avant des artistes originaires des pays auxquels le journal s'intéresse. Ainsi, l'Amérique latine tient une place importante dans le mensuel. L'éditorial «Chávez» (octobre 1999) qui défend la révolution du commandant Chávez au Venezuela, est accompagné d'une peinture du peintre vénézuélien Jacobo Borges intitulée «De ce côté » (1988). En novembre 2003, l'éditorial «Bolivie » sur la révolution bolivarienne est illustré par "Une consolation» (2000) de Fernando Botero, aquarelliste et sculpteur colombien. L'éditorial «Viva Brasil! ( (janvier 2003), qui célèbre la victoire électorale d'octobre 2002 du nouveau président Luiz Inacio Lula da Silva, chef du Parti des travailleurs, est associé à la représentation du tableau «Le Métis » (1954) du peintre Candido Portinari, chef de file du modernisme de la peinture brésilienne. Ce dernier exécute dans un style typiquement brésilien des fresques sur lesquelles figurent ses concitoyens, paysans ou citadins laborieux. En février 2011, face au soulèvement du peuple tunisien contre son pouvoir, Le Monde diplomatique publie des œuvres abstraites d'origine tunisienne Amel Bennys : «Le Grand Oui » (2007), «Passage » (2003), «Oui» (1998), «Attente qui roule vers le grand large » (1999) et «Ensemble, évidemment» (2007). Autre exemple : en mars 2011, la série « Scènes de rues » (2007) du peintre égyptien Mohamed Abla a été choisie pour illustrer un dossier sur les manifestations qui secouent les régimes arabes en place.

\section{L'art au service de la démocratie}

La représentation que donne de l'art Le Monde diplomatique correspond à l'image de l'art dans sa théorie noble : "Idée de l'art - autonomie, valeur absolue, critères esthétiques - et parallèlement idée de l'artiste - exprimant une réalité nouvelle, ou s'exprimant de manière nouvelle, critique de la société et de ses valeurs marchandes [...]» (Cauquelin 1993: 59). En cherchant à créer des «résonances », des « passerelles de sens » entre le lisible et le visuel (Ramonet, 2004), Le Monde diplomatique recrée l'articulation entre art et politique et entre art et 
démocratie. Dans les autres journaux, l'art est relégué aux dernières pages après la politique et l'économie. Les journaux d'information se préoccupent peu de l'art, laissant les revues spécialisées traiter du sujet, alors que dans Le Monde diplomatique, les œuvres peuvent ainsi être vues par un public nombreux en dehors des espaces consacrés à l'art. Ainsi au Monde diplomatique, le langage de l'artiste fait sens hors du champ clos des revues et du monde de l'art. L'art se réapproprie ainsi le réel nié par la critique artistique, l'histoire de l'art et la théorie esthétique. Le Monde diplomatique participe au décloisonnement de l'art. Il a initié une nouvelle conception du rapport entre l'art et le public. Il utilise l'art comme un moyen, un outil de lutte qui dénonce ou sert une cause et non pas comme une fin en soi. Le moyen de communication qu'est le journal permet aux artistes d'agrandir leur rayon d'action. On sait que lié à l'arsenal d'un média et à la liberté d'expression, l'art peut en effet constituer une forme de propagande. Cette forme de communication privilégiée peut éventuellement servir la démocratie en s'adressant au plus grand nombre et en suscitant le débat public. Dans le Monde diplomatique, les œuvres ont un principe actif, l'activisme social, qui permet une prise de conscience du pouvoir politique auquel peut prétendre l'œuvre d'art même si son producteur ne l'a pas conçue dans ce sens. Dans ce cas, c'est le diffuseur - en l'occurrence la rédaction du Monde diplomatique - qui y projette cette prétention.

\section{Conclusion. Le Monde diplomatique : une pratique journalistique en contradiction avec la théorie de sa ligne éditoriale?}

L'usage de l'art dans Le Monde diplomatique participe à sa volonté de résistance, sur le fond et sur la forme. Par son iconographie, le mensuel s'érige à contrecourant de ce qui se fait dans le reste de la presse. Mais le lectorat du Monde diplomatique est-il capable d'appréhender le contenu des ouvres diffusées dans le journal ? Pour répondre à cette question, il faudrait analyser la manière dont l'art est perçu par les lecteurs du Monde diplomatique en faisant une analyse d'opinions ou une analyse critique de l'éducation artistique des lecteurs du mensuel, ce qui n'est pas le propos de cet article. Ce que l'on sait, c'est que le lectorat du Monde diplomatique est instruit: $60 \%$ des lecteurs sont diplômés d'au moins trois années d'étude après le baccalauréat. De plus, c'est un lectorat urbanisé : $72 \%$ des lecteurs du mensuel vivent dans une agglomération de plus de 100000 habitants (plus d'un tiers des lecteurs résident en agglomération pari- 
sienne $)^{10}$. Son lectorat a donc accès aux musées et aux galeries d'art. De la même manière que les textes du journal sont longs et denses, souvent réputés austères, son iconographie contribue à un besoin d'intellectualisation: l'art comme un signe distinctif. Si Le Monde diplomatique entend décloisonner l'art, par certains côtés, sa conception de l'art reste élitiste. Par ailleurs, la démarche du Monde diplomatique est celle d'un choix forcément subjectif. Ce travail d'association d'idées réclame un parti pris, totalement assumé par la rédaction du journal. Mais cela ne va-t-il pas à l'encontre de la volonté d'objectivité dont elle se réclame? On peut s'interroger sur cet engagement journalistique et sur les répercussions de la partialité de l'information. Dans quelle mesure les idées et les choix de la rédaction du Monde diplomatique peuvent-ils être considérés comme relevant de l'information ? Cette dernière ne se trouve-t-elle pas faussée par de trop multiples engagements alors même que Le Monde diplomatique critique les médias et dénonce la manipulation de l'information? Autre piste de recherche : par son contenu et la nature de son iconographie, Le Monde diplomatique s'engage à contre-courant des réseaux médiatiques classiques. Mais respecte-t-il le même engagement par le choix des artistes contemporains publiés ? Ces derniers font-ils partie du réseau international connu de l'art contemporain ? Pour le savoir, il faudrait mener une enquête sur la cote de tous les artistes publiés dans le mensuel, à l'aide par exemple du Kunst Kompass ou d'Artfacts.Net ${ }^{11}$. Par exemple, si on prend en compte les œuvres publiées en première page des douze numéros du Monde diplomatique en 2010, on s'aperçoit que deux artistes font partie du Top 100 de Arffacts.Net ${ }^{12}$. À l'inverse, trois

\footnotetext{
${ }^{10}$ Résultats de l'étude d'audience de la presse magazine AEPM (Audiences et études de presse magazine) pour la période juillet 2009 - mars 2010. Cette enquête est réalisée auprès d'environ 25000 foyers par entretiens à domicile, confiés à trois instituts : IIPSOS, ISL et TNS Sofres. Elle mesure l'audience d'environ 150 magazines français de plus de 100000 lecteurs.

${ }^{11}$ La première mesure est un outil allemand mis au point par une revue financière allemande. Il constitue un palmarès international annuel qui sert de référence aux collectionneurs et investisseurs. Il s'agit d'un classement en fonction de la notoriété et de la réputation de l'artiste. Les artistes sont classés en fonction du nombre d'œuvres possédées par les musées, du nombre d'articles publiés dans la presse spécialisée, et des expositions organisées. Artfacts.Net est un classement travaillant avec une méthodologie un peu similaire mais incluant les artistes disparus.

${ }^{12}$ Paul Klee, dont l'œuvre « Masque de peur » (1932) est publiée en septembre 2010, figure à la onzième place. Alexander Calder, dont l'œuvre « Dents de scie » (1969) est publiée en janvier 2010, se place soixante douzième.
} 
autres artistes ne sont pas du tout référencés ${ }^{13}$. Une enquête complète permettrait de savoir si le journal se détache en privilégiant des artistes méconnus du grand public et des réseaux médiatiques qui font l'art contemporain ou, s'il publie les artistes que le système médiatique tout décrié par Le Monde diplomatique a promus.

\section{Bibliographie}

Abastado C. (1980), Messages des médias, Lyon, Cedic.

Adam J.-M. (1997), «Unités rédactionnelles et genres discursifs : cadre général pour une approche de la presse écrite », in Pratiques $n^{\circ} 94$, Metz.

Balle F. (2009, 14e édition), Médias et sociétés, Paris, Montchrétien.

Berger J. (2004), «Lire, voir » in Le Monde diplomatique 50, Éditions Cercle d'art.

Bourdieu P. (2001), Langage et ponvoir symbolique, Paris, Seuil.

Brand S. (2004), « Lire, voir » in Le Monde diplomatique 50, Éditions Cercle d'art.

Cardon D., Granjon F. (2003), «Les mobilisations informationnelles dans le mouvement altermondialiste», Colloque «Les mobilisations altermondialistes », 3-5 décembre 2003.

Cauquelin A. (1993), L'art contemporain, Paris, Presses universitaires de France, "Que sais-je?».

Charaudeau P. (dir.) (1988), La Presse : Produit, Production, Réception, Paris, Didier Erudition.

Charaudeau P. (1997), Le discours d'information médiatique. La construction du miroir social, Paris, Nathan/INA.

Charaudeau P. (2006), «Discours journalistiques et positionnements énonciatifs. Frontières et dérives ", in Semen, 22.

Charaudeau P. (2005), Les médias et l'information. L'impossible transparence du discours, Louvain-la-Neuve, De Boeck.

Grosse E.U., Seibold E. (1996), «Typologie des genres journalistiques» in Panorama de la presse parisienne, Berlin, Peter Lang.

\footnotetext{
${ }^{13}$ Il s'agit de Malaïka Weber (en novembre 2010), Pape Teigne Diouf (mai 2010) et Jan Voos (avril 2010).
} 
Herman T., Jufer N. (2001), «L'éditorial, « vitrine idéologique du journal »?», in Semen, 13, "Genre de la presse écrite et analyse du discours ».

Jouffroy A. (2004), «Lire, voir» in Le Monde diplomatique 50, Éditions Cercle d'art.

Lacoste Y. (dir.) (1993), Dictionnaire de Géopolitique, Paris, Flammarion.

Lugrin G. (2001), « Le mélange des genres dans l'hyperstructure », in Semen, 13, "Genre de la presse écrite et analyse du discours».

Mouriquand J. (1999), L'écriture journalistique, Presses universitaires de France, "Que sais-je?».

Ramonet I. (2004), «Lire, voir» in Le Monde diplomatique 50, Éditions Cercle d'art.

Rigoulot P. (dir.) (2011), «Le Monde diplomatique passé au crible», in Histoire \& Liberté n ${ }^{\circ} 44$. 